as is termed kakké in Japan, and beri-beri in India and Brazil.

4. Cases are attributable with more or less plausibility to gout, rheumatism, exposure to cold and over-exertion, while in some instances no cause can be assigned.

From these brief considerations it is to be readily seen that this disease may present many different clinical aspects. The most diverse symptoms are encountered, inasmuch as any loss of function or perversion of function attributable to spinal nerves may be the leading feature of an individual case. Subjectively the sensory symptoms, which are usually a pronounced feature, vary from slight numbness or strangeness of feeling to the most exquisite hyperæsthesia or absolute anæsthesia. Ordinarily certain areas will be anæsthetic to touch but extremely painful, constituting the ancesthesia dolorosa of the pedantic. Sensations of pins and needles, creeping, crawling, burning, tingling, and pains of a tearing, boring, twisting, even lightning-like character are the burden of the patient's incessant complaints. The hands and feet may become so sensitive that the weight of clothing, the contact of the hand, or even a breath of air cannot be tolerated. Over the affected area there is tenderness to pressure almost invariably, and this is greatest where the nerve trunk is readily accessible. There is often lessened ability to recognize temperatures in the affected parts, to locate tactile impressions or to distinguish degrees of pressure. The reflexes subserved by the nerves in question are diminished, or more usually make complete default, and this condition of even the knee-jerk in diphtheritic palsy of the ordinary pharyngeal form is a diagnostic sign of great weight (Gowers). Where the condition is due to alcohol and rarely to other causes, a peculiar mental state is noticed in which the moral sense is notably perverted. Such patients will detail with great particularity long experiences which are absurd and impossible upon their face, and their statements in regard to their own condition are quite unreliable.

As the great rule the distribution of the disease is strangely symmetrical, usually involving the lower extremities below the knees and the upper extremities below the elbows. Though no nerve is exempt, the musculo-spiral in the arm and the anterior tibial in the leg are ordinarily selected, causing wrist-drop and foot-drop by the motor paralysis which is one of the primary and most constant, as well as the most important symptoms. The development of paralysis may extend over a period of two or three days or as many months, usually commencing with feebleness in the legs and aching in the calves, followed shortly by implication of the upper limbs and attended by some or all of the sensory disturbances just mentioned. When the respiratory nerves are involved it constitutes a grave feature, and may rapidily lead to a lethal termination. Depending upon the severity of the attack and the rapidity of its onset, trophic disturbances sooner or later appear. In the area of distribution of the affected nerves there may be profuse sweating, producing a miliary or even bullous eruption and a macerated condition of the integument. The dystrophy is manifest in dryness of the skin with cuticular desquamation, falling of hairs, and roughness or even shedding of the nails. The fingers may present the peculiar erythematous, glossy skin characteristic of nerve injury, and mottling and flushing with alternate paleness of the parts is frequently noticed. The muscles are often flabby and plainly wasted. In uncomplicated cases the sphincters are never affected. Electrical examination may develop the reaction of degeneration, but ordinarily something short of the typical formula is found, and only the slightest modifications or none at all may be detected in mild cases, as is readily explained by considering the anatomical peculiarities of the disease.

Depending upon the severity of the individual case, its cause and the possibility of removing that cause, the course of the disease is from three to twenty months and, except nerves of vital importance are implicated, almost always terminates in recovery, which is usually preceded by a recurrence of the sensory disturbances as the nerves regenerate. In the later stages massage, douching and electrical exercise of the muscles to maintain nourishment and to prevent spastic or position deformity, are all of great value, but must be employed with intelligence and moderation. Any break in the integument must be carefully attended to, as healing is slow and a tendency to ulceration prominent.

It is probable that further study of this interesting but wide group of diseased conditions will result in establishing differential features of importance in classification, prognosis and treatment. 167 Dearborn St., Chicago.

SOME REMARKS ON THE RELIEF OF REMOTE NEUROSES, BY THE RESTORATION OF OCULAR EQUILIBRIUM.

BY FRANK ALIPORT, M.D., OF MINNEAPOLIS, MINN.

PROFESSOR OF CLINICAL OPHTHALMOLOGY AND OTOLOGY IN THE UNIVERSITY OF MINNESOTA.

The subject of ocular insufficiencies and oversufficiencies appears to be one of those crazes that occasionally strike the medical profession with cyclonic force. Like cyclones, these crazes are unexpected in their attacks and frightfully devastating while they last. Their careers are shortlived, but they leave many aching hearts behind 
to tell the story of over-credulous patients and over-zealous physicians.

We can learn but little, it is true, without experimentation, but in the name of suffering humanity, there should be some limit placed, beyond which the pitiless drug or knife of the medical man may not leave its mark.

What a multitude of mistakes must be laid to the demon of "reflex" action, who, like a fitful spirit, flits hither and thither in our human anatomy, and ever and anon lays his icy hand upon some one of our organs and claims it for his own. How quickly the medical profession grasps at the hint. Immediately a long chain of diseases is looked upon from a different standpoint. Women are ruthlessly laid upon the operating table and castrated. Children are circumcised; teeth are extracted and noses are cauterized. In short, the abiding place of "reflex neuroses" may be found in any organ of the body, and is liable to an invasion by ignorant zealots. Meanwhile the physician is in a hopeless state of bewilderment. The commonest diseases assume a mysterious appearance, and he fears the presence of the lurking "reflex" behind every muscle, nerve and tissue in the body. It matters not whether a patient complains of his eyes or not, clip, clip go the scissors, and his muscles are severed. It matters not if the child's prepuce utters a note of warning. Off it comes. It matters not whether the woman ever complains of her ovaries. Down she must lie and in a twinkling they are laid on the table beside her, and the surgeon imagines he has achieved a signal triumph, especially if he can show that he has made a great many such operations in a very short space of time.

Why do not physicians conscientiously narrate to us the ultimate results of these cases, upon which they base such wonderful reports? Because, they are ashamed to do so! After they have tried them for a time, and the results are finally forced upon them, they simply quietly lay away their hobby and say nothing about their conclusions. But does it teach them a lesson? Unfortunately no! They are ready for the next visionary dream of some over-zealous enthusiast, and are soon deep in the mysteries of the latest "reflex." Where and when will this insane chasing of a will-of-the-wisp cease? How many of the crippled, the maimed, the halt and the blind must be arrayed before us like the ghosts of Richard's victims, before we are contented to inoculate our practice with a little common sense? The answer is as visionary as a " reflex neurosis." But it is to be hoped that the time is not far off; that the day of our salvation and the salvation of our patients is at hand.

One gleam of sunshine I have been able to extract from the general gloom, has helped me over many a dark place. It is this: Never attack an organ, unless that particular organ is causing dis- tinct uneasiness. If an organ is diseased to an extent sufficient to warrant interference, it will usually make its pathological condition evident. The latest candidate for the approbation of the medical man, is the influence exerted upon the nervous system by insufficiencies and oversufficiencies of the octlar muscles.

The subject of tenotomies for ocular insufficiencies is not a new one. But the subject of graduated tenotomies for ocular insufficiencies, and their effects upon the different organs of the body, is comparatively a new one. Tenotomies have, until recently, meant a complete cutting away of the tendons from the eyeball, and this has referred more particularly-almost exclusive$1 \mathrm{y}$, I may say, to insufficiencies of the internal recti muscles. To Dr. Stevens, of New York, is to be given the credit of having elaborated the subject of graduated tenotomies and advancements, for the relief of reflex and remote diseases, and of having called attention to other varieties of insufficiencies, besides those of the internal recti muscles. To Dr. Stevens must also be given the credit of having systematized this entire subject, and of having given us an exact and intelligent nomenclature. His methods of operating are of the very best, and are of such a nature as to make them valuable to every ophthalmologist. His instruments for operating are as near perfection as can be made, and his phorometer renders the detection of insufficiencies easy and systematic. His claims to cure headaches, head neuralgias, etc., will find sympathy, approbation and acquiescence from all ophthalmologists. But his claim to relieve general chorea, epilepsy, paralysis and other grave and remote neuroses by graduated tenotomies, is so extreme as to warrant the unfavorable verdict rendered by almost the entire profession. His claims were so broad, and his personal statistics so surprising, that it became necessary for the profession either to accept or reject the grounds upon which he stood. Therefore the New York Neurological Society determined to thoroughly and impartially investigate the subject. Accord. ingly in March, I887, with the acquiescence of Dr. Stevens, a commission was appointed, consisting of Drs. Seguin, Starr, Birdsall, Moore, Webster, Dana and Foster (the two latter being appointed by Dr. Stevens himself), to consider the value of Dr. Stevens' treatment. This commission was not compelled to bring in a report at any specified date. This was left to themselves. They were requested to bring in their report whenever they had arrived at a conclusion. They were merely asked to consider the subjects of chorea and epilepsy, these being the subjects upon which Dr. Stevens laid the greatest stress, and in which he claims (without, however, substantiating his claims by intelligent statistics) to cure 50 per cent. of all cases submitted to his charge. A preconcerted plan was agreed upon that was 
undoubtedly fair to all, by which patients were placed under Dr. Stevens' care, and observations of progress taken from time to time. This plan appears to have been carried out honestly by the members of the commission, although claims to the contrary were advanced by Dr. Stevens. The total number of cases sent to Dr. Stevens for treatment was $28 ; 23$ were epileptics; 5 cases of chronic chorea, and all had some form of insufficiency. Fourteen of these cases withdrew for various causes: 5 were unable to attend regularly; 2 were declined by Dr. Stevens because of organic disease; 5 were withdrawn by mutual consent of the nembers of the commission sending the cases and Dr. Stevens, for satisfactory reasons, such as non-attendance, etc. Three were discontinued because they grew worse instead of better under the treatment; hence only i4 remained and became available for observation. These cases were under treatment for periods varying from several weeks to thirty months, and the result showed that no cases were cured. One case was much improved, 5 cases improved, 7 cases unimproved, and the result in one case was unknown. From these results it will be seen that six cases were claimed to be improved; three of these cases were from Dr. Stevens' own private practice, and no exact notes of their previous condition were available. In five cases the improvement was very slight; the sixth case was an epileptic who did about as well under Dr. Stevens' treatment as under the bromide treatment. Some of the patients were made worse instead of better, and in some diplopia and vertigo were developed.

Such in brief was the result of the commission appointed in March, 1887 , and which reported in November, 1889. Their labors appear to have been carried on in a spirit of honesty and fairness. They declare that inasmuch as Dr. Stevens has utterly failed to achieve the great results he claimed, this method of treatment is not worthy to be classed among the principal agents for the cure of chorea and epilepsy. This appears to have been agreed to by even those members of the commission selected by Dr. Stevens himself; Dr. Dana (one of Dr. Stevens' friends), even going so far as to say, that while when he entered the commission he was prejudiced in favor of this method of treatment, the result of his observations had been to make him lose faith in its efficiency. One remarkable fact is, that Dr. Stevens reports to have cured 50 per cent. of the cases of epilepsy and chorea submitted to him in his own private practice. He further claims that the cures materialized promptly; but the commission reports that no cases were cured, and there were practically only three cases that made even doubtful improvement, and the course of improvement in them all was protracted.

The fact of the matter is, Dr. Stevens appears to be enthusiastic and very zealous in his investi- gation of this subject, and has arrived at a point where he claims too much and where his claims have no reasonable hope of being sustained by a fair and impartial examination. If be merely advocated the use of this remedy for headaches, head neuralgias, etc., he would have many endorsers, but as it now stands the breadth of his claims is only equaled by the members of the profession who are his open antagonists. Notwithstanding the fact that the commission was appointed with the acquiescence of Dr. Stevens, and that the commission and Dr. Stevens labored together for a period involving two and one-half years, Dr. Stevens, at the meeting in November, I 889, brings in a protest against the reception of this report by the Society. If he did not wish to work with the commission he should have said so at the start; if he wished to object to the method in which the commission was prosecuting its labors he had ample opportunity to do so. But he accepted the cases that were sent to him (with the exception of the fourteen that were rejected), and treated them presumably to the best of his ability, and it would seem as if he should stand by the results. He even has the poor taste to vent his spleen by unjust and personal allusions to the members of the commission, and it is gratifying to learn that such allusions were passed by in dignified silence by the Society, the personal character of the members of the commission being sufficient guarantee against bigoted animosity cutting any figure in its labors. It appears to me that if such neuroses are dependent upon a lack of ocular equilibrium, Dr. Stevens, with the immense labor that he has put upon the subject, the undoubted information he has acquired, and the great skill that he unquestionably possesses, should be able to achieve exact results, and that successful operations, directed against these conditions, should be positive and rapid in their consequences and not protracted and unsuccessful.

For my part I have little faith in graduated tenotomies. I believe that if a muscle is worth tenotomizing at all, the operation should be complete, and that an absolute separation of the tendon from the eyeball should be brought about. In Dr. Stevens' method the tendon is picked up by a pair of fine forceps and the middle fibres are cut, leaving the two lateral margins of the tendon intact; this looks well in theory, but according to my observation does not materialize in practice. The fibres that are cut are supposed to fall back and become attached to the globe at another point. I do not believe that this is the case. I believe that the fibres fall back during the operation, but in a short time thereafter, in the course of healing and cicatrization, the fibres pull back to their original place of attachment. This statement I have repeatedly verified by observations upon the human being and animals, by first making graduated tenotomies, and, after a while, re- 
opening the conjunctiva and carefully observing the condition of affairs. Dr. Stevens himself, I understand, sometimes finds it necessary to repeat this operation from fifteen to twenty times on a single person. If this is the case, and some of his cases are under observation for two and onehalf years without achieving decided results, it would appear to me that the operation should be condemned and a search made for some new remedy. I believe there is a great deal of nonsense in the subject of ocular insufficiencies and tenotomies, when presented in its most favorable aspect, and it appears to me that almost all of the hundreds of cases that I have examined by Dr. Stevens' phorometer have had some varieties of muscular insufficiency. To summarize then: I believe that errors of refraction and ocular insufficiencies are a fruitful source of headaches, head neuralgia and other neuroses, situated in and about the eyes and head. I believe that such diseases can be remedied by the proper adjustment of glasses and by proper tenotomies. But I do not believe that chorea, epilepsy and other remote neuroses are produced by errors of refraction or by muscular insufficiencies, except as such abnormalities indirectly cause an impairment of the general health, which might in its turn present a favorable soil for the growth of the various neuroses. I have but little faith in graduated tenotomies, and believe that if a tenotomy is indicated at all, the complete operation is necessary.

\section{THE CLINIC.}

\section{A CASE OF NEPHRORRHAPHY.}

A Clinical Lecture delinered at the Jefferson Medical College, Philadelphia.

BY W. W. KEEN, M.D.,

PROFESSOR OF SURGERY

The case I shall show you to-day is rare. It is a case of floating kidney, and I propose to sew it fast to the loin. The patient is a young woman, 3i years old, who was brought to me by Dr. Coleman. Her history is briefly this: Five years ago, while reaching for a package of goods upon a high shelf, she suddenly felt something give way in the right lumbar region and also, as she clearly remembers, heard a distinct snap. She almost fell from the weakness caused by the shock, the package fell to the floor, and she was unable to lift it again because of the pain induced by the effort. From that time to this she has always felt it a labor to lift even comparatively light weights, because of pain in the region of the right kidney, shooting into the groin and thigh of that side. For the last three years she has been practically an invalid. With this there has been at times disturbance of the urinary function, the urine now and then becoming scanty and high colored, but never, as far as observed, containing albumen. Beside this there have been occasionally very marked digestive disturbances, such as diarrhœe, nausea and anorexia. Menstruation is affected but little, but she has thought that at such times and occasionally during the intervals there has been some enlargement of the tumor, which made its appearance after her accident five years ago. If she lies upon her left side, this iump falls with a "thud" to a lower position upon the left side of the abdomen. At the internal border of the tumor I can distinctly feel the pulsation of a large artery, presumably the renal. By a slight manipulation the mass can be pressed forward, and it then falls below the umbilicus and to the left of the middle line. It is like a ship at anchor which swings with the tide to the limit of its hawser. The size of the tumor corresponds to that of the kidney. Its shape is approximately renal but the hilum cannot be distinctly made out. Again, percussion over the right renal region gives a certain amount of resonance, not perfectly clear, but distinctly different from that upon the left side; and there is also a sense of diminished resistance. My colleagues, Profs. Brinton and Parvin, have examined the patient and concurred in my diagnosis.

The kidney is subject to two forms of mobility, which should be carefully distinguished: $I$. The moveable kidney, which, while surrounded by its fatty envelope, is to a certain extent moveable in the space behind the peritoneum;2. The floating kidney, which is provided with a more or less complete meso-nephron, that is a reflexion of peritoneum analogous to the mesentery and mesocolon. A simple moveable kidney may become in time a floating kidney by gradually pushing before it a process of the peritoneum, as does the testis in its descent. Mr. J. Grieg Smith says that the condition is always congenital, but I cannot believe this, for in almost all cases that we see there is a clear history of recent discovery of the tumor, and in very many of an accident to which it is reasonably attributed.

If a patient come to you with such a trouble, what are the means of diagnosis? In nine cases out of ten the patient is a woman, and in nine cases out of ten it is on the right side. Rarely it is the left kidney, and still more rarely both are moveable. Occasionally the patient is a man. moreover, you will find a tumor that can be freely pressed into various regions such as the loin, the umbilical or hypogastric region; you may be able to make out the kidney shape and feel the hilum and possibly the renal artery pulsating, as I can distinctly feel in this case. You will then examine the loin by percussion and by bimanual palpation. But these tests may lead you to a false diagnosis. Three of us have examined this case with care and are reasonably sure of our conclusions; but it is possible we may have mistaken 